\title{
Évolution et caractéristiques de l'institutionnalisation du français en milieu scolaire maltais entre 1850 et 1900
}

Noël Caruana Dingli and Anthony Aquilina

\section{(2) OpenEdition}

Electronic version

URL: https://journals.openedition.org/dhfles/3035

DOI: $10.4000 /$ dhfles.3035

ISSN: 2221-4038

Publisher

Société Internationale pour l'Histoire du Français Langue Étrangère ou Seconde

Printed version

Date of publication: 1 June 1999

Number of pages: $65-81$

ISSN: 0992-7654

\section{Electronic reference}

Noël Caruana Dingli and Anthony Aquilina, "Évolution et caractéristiques de l'institutionnalisation du français en milieu scolaire maltais entre 1850 et 1900", Documents pour l'histoire du français langue étrangère ou seconde [Online], 23 | 1999, Online since 05 September 2017, connection on 10 March 2023. URL: http://journals.openedition.org/dhfles/3035 ; DOl: https://doi.org/10.4000/dhfles.3035

This text was automatically generated on 10 March 2023.

All rights reserved 


\title{
Évolution et caractéristiques de l'institutionnalisation du français en milieu scolaire maltais entre 1850 et 1900
}

\author{
Noël Caruana Dingli and Anthony Aquilina
}

\section{Introduction : quelques points de repère historiques}

1 Avant d'étudier en détail la période qui nous intéresse ici, en l'occurrence la seconde moitié du XIX ${ }^{e}$ siècle, il est utile et méthodologiquement intéressant d'évoquer brièvement l'enseignement du français à Malte avant le XIX ${ }^{\mathrm{e}}$ siècle. Cette démarche permet de mettre le processus d'institutionnalisation de l'enseignement du français dans son contexte historique.

2 Il est possible de distinguer trois moments principaux de l'histoire moderne des îles maltaises susceptibles d'intéresser le didacticien-historien qui fait des recherches sur le français. La première période est l'époque pendant laquelle les Chevaliers de Saint-Jean de Jérusalem, les célèbres Chevaliers de Malte, régnèrent sur l'archipel maltais (1530-1798). Sous les Chevaliers de Malte, le français n'était pas encore une discipline scolaire à part entière mais un art chevaleresque (Pellandra, $1988: 60$ ). Le français était exclu du programme principal du collège de Jésuites de La Valette, le Collegium Melitense, fondé en 1592, et de l'Université de Malte. Certes, il est vrai qu'il existait déjà des démarches administratives qui devaient être faites par tous ceux qui désiraient exercer le métier d'enseignant. En fait, ces candidats devaient présenter une pétition aux autorités compétentes, passer un examen et faire une déclaration de catholicité avant d'être autorisés à enseigner. Il est certain qu'il y avait des enseignants, y compris des enseignants français ou d'origine française, à Malte à cette époque mais les pétitions qui concernent l'enseignement du français brillent par leur absence quasi totale dans les archives maltaises (Borg, $1974: 215-254)^{1}$. Les seules demandes 
d'enseigner le français que nous ayons trouvées furent présentées par des pétitionnaires qui souhaitaient aussi enseigner des matières plus «nobles » telles que le latin et les mathématiques, en plus du français. Nous croyons que les personnes qui voulaient enseigner exclusivement des langues modernes ne se donnaient normalement pas la peine de respecter toutes les formalités bureaucratiques et religieuses requises. Dans ces circonstances, loin d'être institutionnalisé, l'enseignement $\mathrm{du}$ français prenait probablement la forme d'une activité «buissonnière» exercée en dehors des voies de la bureaucratie par des maîtres plus ou moins compétents.

3 Une deuxième période est le bref interlude napoléonien de l'histoire de Malte. La domination de Malte par la France, cette " poussée brève et explosive " selon la terminologie braudélienne (Braudel, 1969 : 45), ne dura que deux ans (1798-1800). A son arrivée à Malte, avant de poursuivre son chemin vers l'Egypte où l'attendaient d'autres combats, le général Bonaparte décréta un certain nombre de réformes éducatives pour Malte. Selon ces réformes, l'enseignement de la langue française allait être introduit (et institutionnalisé) dès l'école primaire. De plus, l'Université de Malte allait être remplacée par une Ecole Centrale. En réalité, à cause des hostilités entre la garnison française d'une part et les insurgés maltais, les Anglais et leurs alliés de l'autre, ces mesures ne furent pas mises en pratique.

Une troisième période de l'histoire moderne de l'enseignement du français à Malte est l'époque de la domination britannique (1800-1964). De notre point de vue, la périodisation didactique ne correspond pas exactement à la chronologie historique ou politique. A titre d'exemple, en ce qui concerne la première moitié du siècle et l'institutionnalisation du français, les années 1800-1838 représentent une période de transition. L'ancien collège de La Valette, rebaptisé « le Lycée », rouvrit ses portes en 1800. On y enseignait les langues suivantes : l'italien, l'anglais, le latin et l'arabe. Pendant les guerres napoléoniennes (mais même après), probablement à cause des mauvais souvenirs qu'avaient laissés les hostilités franco-maltaises des années 1798-1800, mais aussi parce que les Anglais se souciaient plutôt de promouvoir leur langue dans leur nouvelle possession en Méditerranée, le français fut complètement exclu de cet établissement scolaire pendant une quarantaine d'années. Pendant cette période, la « survie » de l'enseignement du français à Malte fut assurée par des écoles privées. Il s'agissait d'écoles plus ou moins réputées qui étaient souvent dirigées par des étrangers, y compris par des Anglais qui, même en temps de guerre, n'hésitaient pas à inclure le français dans leurs programmes ! Le Journal officiel de Malte des années 1812-1838 publia 21 offres d'enseignement du français sur un total de 48 annonces « scolaires » parues (soit $44 \%)^{2}$.

5 A Malte, deux textes officiels annoncent le début historique de l'enseignement du français dans le secteur public à l'époque coloniale : le rapport des commissaires royaux J. Austin et G. Cornwall-Lewis, envoyés à Malte en 1836 (Austin, Cornwall-Lewis, 1838) et le Statut Fondamental du Lycée et de l'Université de Malte qui fixa pour la première fois le salaire d'un professeur de français au Lycée (£ 50 par an). 


\section{L'enseignement du français à Malte pendant la seconde moitié du XIX'e siècle}

\section{Les années 1838-1880}

Plutôt que l'année 1850, c'est 1838/1839 qui marque un tournant décisif et le début de l'institutionnalisation du français à l'école publique à Malte.

7 Les raisons qui expliquent l'entrée du français dans les établissements scolaires d'Etat à Malte sont au moins au nombre de cinq :

8 L'effondrement de l'Empire. Presque quarante ans après la capitulation et le départ des Français de Malte, il était temps d'oublier le passé.

9 L'utilité pratique de la langue française. Le français était une langue qui était bien connue par une section de la population en Grande-Bretagne. Il ne faut donc pas exagérer les conséquences négatives des guerres sur l'emploi des langues. A ce point, il n'est peut-être pas inutile d'examiner un peu les rapports entre les guerres et les langues. Pendant les guerres napoléoniennes, à Malte, comme dans d'autres pays, des officiers britanniques n'hésitaient pas à employer le français pour parlementer et communiquer avec leurs ennemis. Sir Alexander Bail, commandant des forces navales britanniques, alla jusqu'à adresser des lettres en français à Vincenzo Borg, l'un des chefs des insurgés maltais qui combattaient contre les Français ! Autre fait " surprenant »: des Maltais qui avaient beaucoup souffert après l'arrivée des Français à Malte envoyèrent des pétitions rédigées en français aux autorités britanniques et maltaises !4

10 L'émigration massive vers les territoires français d'Afrique du Nord commença peu après le début de la colonisation britannique. Il était donc utile de promouvoir l'enseignement du français à Malte.

$11 \mathrm{Au} \mathrm{XIX}^{\mathrm{e}}$ siècle, on assista à une demande croissante pour la création de filières nouvelles et l'enseignement de disciplines modernes. Malte ne pouvait pas échapper indéfiniment à cette tendance européenne.

12 Après cette première apparition prometteuse dans l'enseignement public, le français manqua une occasion qui lui aurait permis de consolider sa position par rapport au latin au sein du lycée de la capitale maltaise. En 1842 le recteur de l'Université, le révérend T. O'Malley élabora un projet (O'Malley, 1842) et proposa de diminuer l'importance accordée à la langue latine dans cet établissement. Cependant, les autorités refusèrent de donner une suite favorable à cette initiative et le projet n'aboutit pas.

13 Avant d'aller plus loin, il est intéressant de présenter des caractéristiques du paysage éducatif maltais avant 1880 . Pendant cette période, la situation au Lycée devint assez chaotique : bien qu'il y existât des cursus, il n'y avait pas encore de filières précises. Les élèves étaient complètement libres dans le choix des matières qu'ils étudiaient (ce qui faisait plus d'une centaine d'options possibles !). En fait, ils avaient vraiment l'embarras du choix. Par conséquent, tout en étant institutionnalisé et reconnu comme discipline légitime, le français n'était qu'une matière parmi d'autres. Il n'était pas moins institutionnalisé que les autres matières qu'on enseignait dans cette école secondaire car ces matières étaient exactement dans la même situation. 

$\mathrm{du}$ français car elle se limita à réviser le barème des salaires des professeurs. Par contre, un rapport qui eut des conséquences importantes fut celui du commissaire royal P. J. Keenan, publié en 1880. M. Keenan fut envoyé à Malte pour faire une enquête sur l'état de l'éducation. Comme nous l'avons déjà dit, certains aspects du système éducatif maltais étaient préoccupants. Les autorités coloniales ne pouvaient surtout pas tolérer une situation où la langue coloniale était beaucoup moins répandue que la langue italienne qui avait à la fois le statut de langue de culture, langue administrative et langue des tribunaux à Malte. Les effets du « Keenan Report » se feraient encore sentir même une cinquantaine d'années après sa publication. assista à des cours de français au Lycée de La Valette en 1878/1879. Son témoignage précieux nous révèle comment on y enseignait le français à cette date :

L'enseignement de la langue française s'étale sur trois ans. M. Cherpit, le professeur, est suisse. Il a commencé son stage probatoire en octobre 1876 et sa situation n'a pas changé depuis cette date. Il y a 73 élèves qui étudient le français. Leur présence au cours est très irrégulière (...) Dans la classe de français je m'attendais à trouver la culture littéraire générale en position privilégiée, ou bien une disposition très marquée pour les études commerciales. Les critères que j'avais retenus pour la vérification de mes hypothèses étaient respectivement le latin et la comptabilité. Mes prévisions, cependant, étaient loin d'être correctes. Des 73 élèves qui suivent des cours de français, 16 apprennent le latin et 5 apprennent la comptabilité ; 68 apprennent l'italien, 56 font de l'anglais et 55 étudient 
l'arithmétique. Le programme comprend la grammaire, la composition et la traduction (a) français-italien et (b) italien-français. Comme dans toutes les classes, une place de première importance est réservée à l'italien qui est la langue d'enseignement. Les exercices de traduction étaient bien faits mais, pendant l'exercice de lecture, l'accent et la prononciation laissaient beaucoup à désirer. Dans la classe supérieure, les élèves lisent les Morceaux Choisis de Buffon et ils font des traductions français-italien; et puis, inversement, ils traduisent Le Mie Prigioni de Silvio Pellico en français. Dans la classe d'italien, le sujet de l'exercice mensuel de composition est donné par le professeur. Dans les classes de français, par contre, chaque élève choisit son propre sujet. Les exercices contenaient beaucoup de fautes mais $M$. Cherpit a su en profiter pour faire des critiques et des remarques très pertinentes. Un jour que je me trouvais là, un petit garçon de la classe d'arithmétique est arrivé quand la leçon de français était à moitié terminée... (Keenan, 1880 : 76-77 ; notre traduction de l'anglais.) royal ne s'en prend pas à ce professeur consciencieux. Ce qui constituait un problème, c'était surtout le manque d'organisation (absence de filières, absentéisme, etc.). Grâce au témoignage précieux de $\mathrm{M}$. Keenan, il est évident que la méthode de grammairetraduction battait son plein dans ce lycée. En réalité, il n'en allait pas autrement dans les autres écoles maltaises à cette époque. L'enseignement du français au Lycée se faisait toujours par le moyen de l'italien, c'est-à-dire par l'intermédiaire d'une langue qui n'était pas la langue maternelle de la plupart des élèves. qui allait durer jusqu'aux années 1930 à Malte : la «Question de la Langue». Selon L.-J. Calvet, il y a une corrélation très étroite entre les "guerres des langue» et le plurilinguisme. S'il y a guerre linguistique, c'est parce qu'il y a plurilinguisme : «... le plurilinguisme (...) est à l'origine du conflit. Non pas motif unique de la guerre mais condition sine qua non de sa tenue.» (Calvet, 1987 : 32). Etant donné la coexistence de l'anglais, de l'italien et du maltais, cette condition était bien remplie dans le contexte maltais.

Pendant la seconde moitié du XIX siècle il y avait de hauts fonctionnaires qui étaient responsables du système éducatif maltais et qui connaissaient le français. A titre d'exemple, le directeur de l'Instruction primaire, le chanoine Paolo Pullicino (1815-1890) suivit des cours à la Sorbonne et on le complimenta sur sa connaissance de la langue française. Mais le chanoine Pullicino était responsable des écoles primaires, secteur où il était hors de question d'introduire l'enseignement précoce du français. Francesco, ou mieux «Napoléo », Tagliaferro (le surnom mérite d'être noté) était, lui, directeur de l'Instruction publique. Il avait donc la responsabilité des écoles secondaires. M. Tagliaferro avait également poursuivi ses études de mathématiques et de physique à Paris. Cependant, dans le climat délicat de la «Language Question», il aurait été absurde de vouloir compliquer encore plus le débat en proposant de mettre le français sur un pied d'égalité avec les autres langues connues à Malte ${ }^{5}$.

Sigismondo Savona (1837-1905) fut nommé directeur de l'Instruction publique en 1880. Il faisait partie du camp des «réformistes» qui voulaient promouvoir l'enseignement de l'anglais et du maltais (par opposition aux «anti-réformistes» qui défendaient les intérêts de l'italien). En essayant de suivre les recommandations du «Keenan Report», Sigismondo Savona, tout en étant un anglophile déclaré, n'alla pas jusqu'à exclure complètement l'italien. Une telle mesure extrême, qui aurait sans doute nettement 
amélioré la position du français dans la hiérarchie des légitimités linguistiques à Malte, était impensable dans le contexte de l'époque.

Pendant la "Question de la Langue», on continua à enseigner le français et sa position plutôt dominée tourna même à son avantage car il était à l'abri des "persécutions » et des « excommunications » qui frappèrent une langue dominante comme l'italien. Grâce aux réformes entreprises par le directeur Savona, le français consolida sa position dans le système éducatif maltais. Ainsi, il fut décidé qu'à partir de l'année 1881 il existerait deux types de cursus au Lycée de la Valette (et ce type d'organisation fut aussi adopté par des écoles privées), à savoir, (a) le Cours Moderne où étaient admis des élèves qui souhaitaient suivre une carrière dans l'armée, dans le commerce, dans l'administration, etc., (b) le Cours Classique qui accueillait les futurs étudiants universitaires, ceux qui se destinaient aux professions libérales. A partir de cette date, le français devint une matière obligatoire pour les élèves du Cours Moderne alors que les élèves du Cours Classique devaient obligatoirement apprendre les langues classiques. Il est vrai que l'anglais et l'italien étaient obligatoires pour tous les élèves mais, même avant la réforme, beaucoup d'élèves du Lycée (67 \% en 1865) apprenaient déjà ces deux langues ; ce qui n'était pas le cas pour le français. En fin de compte, quand on compare les chiffres officiels des années 1878-1880 à ceux des années suivantes, on s'aperçoit qu'il y eut surtout une nette augmentation (de l'ordre de 20 à $25 \%$ ) du nombre d'élèves de français dans cette école (Savona, $1883: 21-24)$.

Grâce à ces réformes, la position du français en tant que discipline scolaire fut à la fois assurée et renforcée alors que la position d'autres langues telles que l'allemand, le grec et l'arabe devint précaire. Quant à l'italien, il perdit progressivement du terrain (Hull, 1993 : 80-81). De cette façon, le bilan, en ce qui concerne les conséquences de la "Question de la Langue» sur l'institutionnalisation de l'enseignement du français, est assez positif. Après sa " consécration » comme matière obligatoire pour tous les élèves du Cours Moderne, à la fin des années 1880 on essaya de l'introduire dans les classes inférieures et de l'enseigner ainsi aux futurs élèves du Cours Moderne. De plus, le français finit par devenir un critère de sélection aux examens de fin d'année du Lycée pendant le premier quart du XXe siècle. On organisa aussi des cours du soir de français alors que d'autres langues importantes telles que l'anglais et l'italien furent longtemps exclues de ce type d'enseignement.

\section{Autres aspects de l'institutionnalisation de l'enseignement du français}

L'institutionnalisation du français en milieu scolaire maltais à l'époque victorienne se fit, d'un point de vue méthodologique, par la grammatisation (le mot est de Renée Balibar), la traduction et l'étude des grands auteurs français. Cela est vrai non seulement dans les écoles publiques mais aussi dans les écoles privées. Nous nous limiterons ici à donner un seul exemple d'une grammatisation extrême, mais prévisible, lorsqu'on tient compte du principe d'émulation si cher aux Jésuites. L'extrait suivant est tiré du programme des « exercices publics » qui eurent lieu du 17 au 19 juillet 1849 au Collège Saint-Paul, établissement dirigé par les Jésuites :

( $2^{\mathrm{e}}$ journée)

$\mathrm{II}^{\mathrm{e}}$ Classe de français 
Messieurs Cassia Vincenzo, Dalmas Giovanni, Dedomenico Ottone, Degiorgio Pierpaolo, Feraldi Francesco, Ferro Alfredo, Mamo Federico, Nicosia Giuseppe, Sciortino Adolfo, Tagliaferro Alessandro, Tagliaferro Emmanuele

Expliqueront les fables de Chambaud, et feront les exercices sur les verbes de la grammaire de Goudar

Réciteront et expliqueront quelques fables de La Fontaine ainsi qu'une scène des Plaideurs de Racine.

TROISIEME JOURNEE

Première Classe de Français

Messieurs

Albanese Giuseppe, Albanese Antonio, Cachia Salvatore, Ferro Guglielmo, Ittar Agapito, Le Brun Giacchino, Mifsud Pasquale, Naudi Agostino, Parnis Guglielmo, Zammit Giorgio

Proposeront de réciter toute la grammaire composée par Messieurs Noël et Chapsal - de traduire en italien tout le Télé-maque et n'importe quel autre livre français - de réciter et de traduire quelques morceaux choisis tirés des tragédies de Racine - de traduire en français quelques morceaux choisis de Segneri et Monti - de répondre en français à des questions sur tout le contenu des dialogues de Perrin » (Saggio degli studi..., 1849 : 6-7 ; notre traduction de l'italien).

Cette tendance, même sous une forme moins extrême, était, en fin de compte, inévitable. Comme l'ont très bien expliqué des didacticiens comme Henri Besse et Christian Puren (Besse, 1985 : 10-11 ; Puren, 1988 : 45), pour faire face à la prédominance et à la tradition glorieuse des langues anciennes, les enseignants des langues vulgaires avaient intérêt à «mimétiser» l'enseignement institutionnalisé des langues anciennes en mettant l'accent sur la grammaire (cela prouvait qu'il s'agissait d'une langue aussi « sérieuse » que le latin et le grec), la traduction, caractéristique incontournable de l'enseignement des langues mortes, et des textes ayant des sujets qui s'inspiraient de l'Antiquité. Dans cette stratégie pour se faire reconnaître, on fit appel, à Malte, à des « institutions »: les ouvrages de Noël et Chapsal, Larive et Fleury, etc.

Cette institutionnalisation $d u$ français se caractérisait aussi par un certain cosmopolitisme des manuels de français. A cette époque, on enseignait le français à Malte au moyen d'ouvrages français, italiens et anglais. Parmi les auteurs de manuels à succès on peut citer Fasnacht, Goudar, Ollendorff et Pellissier. Ironiquement, cette institutionnalisation fut parfois mise en place dans des écoles secondaires maltaises grâce à des manuels destinés à des élèves latinisants ou de l'école primaire en France. Il est donc possible de parler d'une loi " tendancielle ", d'un déplacement du bas (le niveau primaire en France) vers le haut (l'enseignement secondaire à Malte). En d'autres termes, il s'agissait d'utiliser des livres scolaires à un niveau, dans un contexte et pour des objectifs différents de ceux pour lesquels ils avaient été élaborés. A Malte, contrairement à la France des années 1880, « il (ne) fallait (pas) apprendre à écrire à tous les petits Maltais» (nous nous inspirons évidemment ici du titre du livre bien connu sur la grammaire scolaire par André Chervel (Chervel, 1977) car même en ce qui concerne l'école primaire, l'obligation scolaire pour tous à Malte ne fut définitivement établie qu'en 1946. Si, à la fin du XIX siècle en France, on pouvait parler d'un enseignement " républicain, scolaire, grammatical et primaire» (Balibar, 1985), l'enseignement institutionnalisé du français à Malte pendant cette période était colonial, scolaire, grammatical et secondaire. 

souvent considéré comme une " option » pour laquelle il fallait payer un supplément. A une époque où l'analphabétisme était très répandu à Malte (en 1871, 80 \% des 142000 habitants de l'archipel maltais étaient illettrés ; Hull, 1993 : 32), l'enseignement du français apparaît comme un luxe qui était inaccessible à la très grande majorité des habitants. Signalons, toutefois, que certains enseignants enseignaient le français dans plus d'un seul établissement. Les élèves qui avaient la chance d'avoir été admis au Lycée public avaient donc la possibilité de suivre les cours de ces professeurs compétents qui exerçaient aussi leurs fonctions dans des écoles beaucoup plus chères. A titre d'exemple, Achille Segond et Valentin Tonna-Barthet, tout en étant professeurs de français au Lycée, étaient aussi recherchés par des écoles rivales comme le Collège Sainte-

41 Catherine et le Collège Flores. Enfin, le fait que le français fût considéré comme un luxe est confirmé par son exclusion des programmes de l'enseignement gratuit : ni la « Charity School ", ni la "Orphan School ", ni l'école gratuite dirigée par les pères Augustiniens ne dispensaient d'enseignement du français.

\section{Conclusion : et après ?}

L'examen du baccalauréat (« Matriculation examination ») fut créé à Malte en 1882. Comme c'était prévisible, pendant plusieurs années, une place de première importance fut réservée à l'anglais et à l'italien. Le français fut exclu de cet examen pendant vingtcinq ans mais, à partir de 1907, les étudiants maltais avaient également la possibilité de passer leur baccalauréat de français. Il s'agit d'une date importante dans l'histoire du français à Malte où, après tout, le français a toujours été une langue étrangère pour la

Documents pour l'histoire du français langue étrangère ou seconde, 23 | 1999 
majorité des habitants. Il n'est pas inintéressant de signaler, dans cette optique, que cette date est même antérieure à la création d'un baccalauréat de grec qui fut instauré en 1922. De même, le baccalauréat de maltais, langue maternelle de la plupart des Maltais, n'existe que depuis 1936!

Le début de l'institutionnalisation de l'enseignement du français à Malte remonte à la première moitié du XIX siècle. Pendant la seconde moitié du siècle, le français continua à faire des progrès, à consolider sa position et à s'implanter plus fermement dans le paysage éducatif maltais par rapport à d'autres langues modernes ou classiques. En réalité, encore une fois, ce sont les années 1880 et non pas la fin du siècle, qui constituent un tournant décisif.

Dans les années 1930, l'histoire institutionnelle du français dans le système scolaire public connut d'autres rebondissements surprenants. Les autorités britanniques, inquiètes à cause des progrès trop lents de la diffusion de la langue coloniale à Malte après plus d'un siècle de domination, se rendirent compte qu'elles pouvaient se servir de la promotion de la langue française afin de diminuer encore plus l'importance de l'italien. Ainsi la Commission royale de 1931 recommanda de favoriser l'enseignement du français au dépens de l'italien. Au Lycée de La Valette, le français devint l'une des matières dites « indispensables » : si un élève n'était pas reçu à l'examen de n'importe laquelle de ces matières il n'était pas promu à la classe supérieure. En 1938 on alla encore plus loin car on décida que tout élève du Cours Moderne qui avait réussi à son examen de fin d'année de français serait promu même s'il avait échoué à l'examen d'italien. Enfin, au lendemain de la deuxième Guerre Mondiale, le français accéda au niveau institutionnel le plus élevé : le niveau universitaire. En 1946, M. Gaston TonnaBarthet (voir aussi supra) fut le premier enseignant de français à être nommé à l'Université de Malte. Le français avait donc fait des progrès très significatifs depuis le début du XIX ${ }^{e}$ siècle mais on en était encore très loin de l'émergence d'un champ maltais de FLE car ce phénomène à Malte ne date que des années 1970.

\section{BIBLIOGRAPHY}

Archives Nationales (Malte), fonds du Ministère de l'Education, dossier $n^{\circ} 2 / 250$.

BALIBAR, Renée (1985), «L'Ecole de 1880. Le français national : républicain, scolaire, grammatical, primaire ", in : Antoine, Gérald, Martin, Robert (dir.). Histoire de la langue française (1880-1914).

Paris, Ed. du CNRS : 255-293.

BALIBAR, Renée (1985), L'institution du français. Essai sur le colinguisme des Carolingiens à la République.

Paris, P. U. F.

BESSE, Henri (1985), « Remarques sur le statut de la didactique des langues étrangères dans le champ des sciences humaines et sociales ", Bulletin de l'ACLA : 7-27.

BORG, Vincent (1974), «Development in éducation outside the Jesuit Collegium Melitense ». Melita

Historica, VI, $3: 215-254$. 
BRAUDEL, Fernand (1969), Ecrits sur l'histoire. Paris, Flammarion.

CALVET, Louis-Jean (1987), La guerre des langues et les politiques linguistiques. Paris, Payot.

CHERVEL, André (1987), ... et il fallut apprendre à écrire à tous les petits Français : histoire de la grammaire scolaire. Paris, Payot.

CLAVÈrES, Marie-Hélène (1988), « Naître ou devenir : A propos de la formation des enseignants au dix-neuvième siècle ", Cahiers de l'Asdifle $n^{\circ} 1$, Actes des $2^{\text {èmes }}$ rencontres des 1 et 2 septembre 1988 («Formation en Didactique des langues étrangères ») : 21-37.

Convitto San Giuseppe Valletta, 9 et 10 Agosto 1867. Saggio degli studii Anno V. (1867), Malte, Tip. AngloMaltese.

CORNWALL-LEWIS, George, Austin, John (1838), Reports ofthe Commission Appointed to Inquire into the Affairs ofMalta. Londres, Her Majesty's Stationery Office.

(The) Daily Malta Chronicle (1898), 26 septembre, p. 2.

Fundamental Statute ofthe University of Malta. (1838), Malte, Government Press.

GALEA, Michael (1991), Mons. Dr. Paolo Pullicino (1815-1890). Malte, Vexillina Press.

HULL, Geoffrey (1993), The Malta Language Question. A Case Study in Cultural Imperialism. Malte, Said International.

KEENAN, Patrick (1880), Report upon the educational System of Malta. Londres, H. M. Stationery office.

L'Ordine ; giornale politico, religioso, letterario (1849-1902), Malte.

Malta Blue Book (1821-1938), Malte.

Malta Royal Commission 1931 Report. (1932), Londres, His Majesty's Stationery Office.

O'MALLEY, Thaddeus (1842), Progetto di Studi per VUniversità e il Liceo di Malta. Malte, Stamperia del Governo.

PELLANDRA, Carla (1988), « Enseigner le français en Italie aux XVII ${ }^{\mathrm{e}}$ et XVIII ${ }^{\mathrm{e}}$ siècles », Le français

dans le monde, $218: 58-62$.

Prospectus. Flores s Collège, Valletta (1886), Malte.

PUREN, Christian (1988), « 'Au commencement fut le verbe' : méthodologie directe, professionnalisation et formation ", Cahiers de l'Asdifle $\mathrm{n}^{\circ} 1$, Actes des $2^{\text {èmes }}$ rencontres des 1 et 2 sept. 1988 («Formation en Didactique des Langues Etrangères »), 39-52.

Report of the Commission appointed by His Excellency the Governor to enquire into the state of public instruction, as taught in the Lyceum and the Primary Schools, Malta Government Gazette (1865), 2229 :

234-240.

Saggio degli studi nel Convitto di San Paolo in Città Notabile nei giorni 17, 18 e 19 luglio 1849 (1849), Malte. SAVONA, Sigismondo (1883), Report on the educational intimions of Malta and Gozo. Malte, Government Printing Office.

Statuto del Collegio Santa Caterina eretto in Valletta sotto il Patronato dell'Eccellenza Reverendissima di Monsr. Fra Gaetano Pace Forno Arcivescovo di Rodi, e Vescovo di Malta (1864), Malte, Tipografia AngloMaltese.

VADALÀ, R. P. M. (1911), Les Maltais hors de Malte : étude sur l'émigration maltaise. Paris, A. Rousseau. 


\section{NOTES}

1. Voir aussi les pétitions (Suppliche 1769-1800) conservées aux archives de l'Archevêché de Malte (A. A. M.).

2. Voir Il Giornale di Malta / Gazzetta del Governo di Malta I Malta Government Gazette, années 1812-1838.

3. Musée National des Beaux-Arts (Malte), « Bail Papers » (manuscrits de la « Fondation Gollcher »).

4. Archives Nationales (Malte), NA(M) 92/04 cartons 606, 616, 627, 628 (1798-1800).

5. A l'époque coloniale, les manifestations publiques de francophilie à Malte avaient surtout une fonction symbolique. Le chant de la Marseillaise, le déploiement de drapeaux tricolores et des cris de «Vive la France ! » dans les rues, au théâtre, etc. servaient, de temps en temps, à protester contre le colonialisme britannique.

6. Voir l'annonce parue dans le journal maltais L'Ordine $\mathrm{n}^{\circ} 481$ (3 sept. 1858, p. 5204).

7. Malta Blue Book, années 1880-1903.

8. Témoignage d'un ancien élève octogénaire. Archives Nationales (Malte), fonds du Ministère de l'Education, dossier n² 2/50 ; Malta Blue Book, années 1891-1938.

9. Archives Nationales (Malte), fonds du Ministère de l'Education, dossier $n^{\circ} 2 / 50$; Malta Blue Book, Année 1891-1938.

10. Voir l'annonce parue dans le journal maltais L'Ordine, $n^{\circ} 1455$ et 1456 (les 3 et 10 juillet 1894), s. p.

11. Ibid., $\mathrm{n}^{\circ} 684,8$ août 1862 , p. 3.

\section{AUTHORS}

\section{ANTHONY AQUILINA}

Université de Malte 